\title{
Normativität und Bayesianismus
}

\author{
Stephan Hartmann und Ludwig Fahrbach
}

Das Thema dieses Bandes ist die Frage, ob die Wissenschaftstheorie eine normative Disziplin ist. Zunächst überrascht die Frage, denn für viele Wissenschaftstheoretiker ist die Antwort ein klares „Ja“; sie halten es für einen Allgemeinplatz, dass die Wissenschaftstheorie ein normatives Unternehmen ist.

Bei genauerem Hinsehen stellt sich jedoch heraus, dass die Frage unterschiedliche Interpretationen zulässt, die einzeln diskutiert werden müssen. Dies geschieht im ersten Abschnitt. Im zweiten Abschnitt suchen wir nach möglichen Erklärungen dafür, warum die Wissenschaftstheorie bisher bei dem Projekt, eine allseits akzeptable Methodologie wissenschaftlichen Schließens zu formulieren und zu begründen, so wenig Erfolg hatte. Eine mögliche Erklärung für den ausbleibenden Erfolg ist der Partikularismus, wonach Methoden keine allgemeine, sondern nur eine lokale, bereichsabhängige Gültigkeit haben. Im dritten Abschnitt wollen wir an Hand des Bayesianismus zeigen, dass die Methodologie doch nicht so schlecht da steht, wie es zunächst den Anschein hat. Der Bayesianismus ist für viele Wissenschaftstheoretiker der aussichtsreichste Kandidat für eine allgemeine Theorie induktiven Räsonierens. Wir besprechen seine Vorzüge, stellen aber auch dar, welche Konzessionen er an den Partikularismus machen muss.

\section{Normativität in der Wissenschaftstheorie}

Wissenschaftstheoretiker haben Wissenschaft auf viele unterschiedliche Weisen bewertet und kritisiert. Zum Beispiel haben sie Überlegungen zu der Frage angestellt, welche Dinge die Wissenschaft erforschen sollte. Auf allgemeiner Ebene behandelt beispielsweise Philip Kitcher (2001) solche Fragen. Er untersucht, wie erreicht werden kann, dass die Wissenschaft „wohlgeordnet" ist, d.h. wie auf gerechte und demokratische Weise entschieden werden kann, welche Dinge die Wissenschaft erforschen sollte und auf welche Weise Ressourcen wie Geld und Positionen sinnvoll über die verschiedenen wissenschaftlichen Fächer und Projekte verteilt werden können. Eine andere Frage über die Organisation der Institution Wissenschaft behandelt James R. Brown (2000). Er kritisiert die Tendenz, dass immer mehr Forschung von privaten Unternehmen durchgeführt und damit gesellschaftlicher Kontrolle entzogen wird. Dies sind nur zwei Beispiele dafür, wie Wissenschaftstheoretiker Kritik an der der Wissenschaft üben.

Doch sind diese Formen der Kritik an der Wissenschaft nicht diejenigen, die wir im Folgenden untersuchen wollen. Nach unserem Verständnis der Eingangsfrage, ob die Wissenschaftstheorie eine normative Disziplin ist, geht es in ihr nicht um soziale, politische oder organisatorische Probleme der Wissenschaft. Vielmehr verstehen wir die Eingangsfrage so, dass sie sich auf die Aufgabe der Wissenschaftstheorie bezieht, die Relation der Bestätigung zwischen Beobachtung und Theorie zu untersuchen und Prinzipien der rationalen Theoriewahl zu formulieren und zu begründen, also Prinzipien, um auf der Grundlage der jeweils vorliegenden Beobachtungen und Daten zwischen verschiedenen Theorien (bzw. für oder gegen die Akzeptanz einer einzelne Theorie) auf rationale Weise zu entscheiden. Die zugehörige wissenschaftstheoretische Disziplin ist die Methodologie. Sie formuliert und 
untersucht Systeme von Prinzipien für das Akzeptieren und Ablehnen von wissenschaftlichen Theorien und sucht nach möglichen Begründungen für solche Prinzipien. ${ }^{1}$

Die erste Möglichkeit, wie die Eingangsfrage („Ist die Wissenschaftstheorie eine normative Disziplin?“) verstanden werden kann, ist die folgende:

(1) Gibt es objektiv gültige Prinzipien der rationalen Theoriewahl?

In einem Augenblick werden wir zu dieser Frage ein paar Bemerkungen machen, doch zuvor wollen wir überlegen, auf welche Weise die Eingangsfrage noch verstanden werden kann.

Zunächst scheint es offensichtlich zu sein, dass der Untersuchungsgegenstand der Methodologie (also die Prinzipien der rationalen Theoriewahl) eine normative Natur hat. Die Prinzipien haben zum Inhalt, wann die Akzeptanz einer Theorie rational ist, wie die Wissenschaftler induktiv schließen sollten, und so weiter. Das sind Aussagen darüber, was der Fall sein sollte.

Darauf könnte man erwidern, dass der Eindruck täuscht und die Prinzipien, genauer betrachtet, gar nicht normativ aufzufassen sind. Viele Wissenschaftstheoretiker und Erkenntnistheoretiker vertreten eine instrumentelle Auffassung, wonach Prinzipien und Methoden nur Mittel sind, um gewisse epistemische Ziele zu erreichen, etwa das Ziel, wahre Theorien zu finden. Die Methodologie untersucht dann nur, ob die Prinzipien und Methoden als Mittel effektiv sind, um die jeweiligen epistemischen Ziele zu erreichen. ${ }^{2}$ Sie untersucht nur „hypothetische Imperative“. Zwar sind „hypothetische Imperative“ immer noch Imperative, denn sie gelten für all diejenigen Fälle, in denen das Subjekt (etwa der Wissenschaftler) die jeweiligen epistemischen Ziele tatsächlich hat und macht für diese Fälle Sollensaussagen, aber die Methodologie kann dennoch rein deskriptiv aufgefasst werden, denn sie untersucht nur die Behauptung, ob die Prinzipien jeweils faktisch effektiv sind, um die epistemischen Ziele zu erreichen; sie hat nicht zum Ziel, Imperative aufzustellen oder Sollensaussagen zu machen, so diese Erwiderung.

Doch selbst wenn es stimmt, dass die Methodologie rein deskriptiv verstanden werden kann, so ist es doch nur ein kleiner Schritt für den Methodologen, die Prinzipien herzunehmen und das faktische Verhalten der Wissenschaftler mit den Prinzipien zu vergleichen, auf Übereinstimmung oder Abweichung hin zu überprüfen und dementsprechend zu bewerten. Wenn ein Methodologe glaubt, er habe ein gewisses Maß an Wissen über seinen Gegenstand, also diese oder jene Einsicht über die Rationalität von Theorienakzeptanz gewonnen, dann liegt es für ihn nahe, dieses methodologische Wissen anzuwenden und die konkrete Wissenschaftspraxis mit Hilfe seiner methodologischen Einsichten zu bewerten und zu kritisieren.

Dies führt zu einer zweiten Frage:

(2) Bewerten und kritisieren Wissenschaftstheoretiker die Wissenschaft, oder halten sie sich mit methodologischer Kritik zurück?

In dieser Frage geht es um das faktische Verhalten der Wissenschaftstheoretiker. Wir können aber auch nach der Berechtigung zu solchem Verhalten fragen:

\footnotetext{
${ }^{1}$ Statt „Prinzip“ verwenden wir auch das Wort „Methode“. Das Wort „Methodologie“ ist bekanntlich mehrdeutig. Es kann entweder eine Menge von Methoden bedeuten - dann spricht man von „einer Methodologie“ - oder es kann die philosophische Disziplin bedeuten, die sich mit der Formulierung und Begründung von wissenschaftlichen Methoden und Prinzipien und der Aufstellung von Methodologien im ersten Sinn beschäftigt - dann spricht man von „der Methodologie“.

${ }^{2}$ Quine (1986, S. 664-665), Sankey (2000), u.v.a. Siehe dazu den Beitrag von Bernward Gesang.
} 
(3) Sind die Wissenschaftstheoretiker dazu berechtigt, die Wissenschaft methodologisch zu kritisieren?

Dies führt schließlich zu der Frage, ob Wissenschaftstheoretiker sogar die Pflicht haben, sich in die Wissenschaft einzumischen:

(4) Sind die Wissenschaftstheoretiker dazu verpflichtet, die Wissenschaft methodologisch zu kritisieren?

Die letzte Frage kann als zweite Interpretation der Ausgangsfrage verstanden werden. Wir wollen nun alle vier Fragen nacheinander behandeln. Wir nennen jemanden, der Frage (1) mit „Nein“ beantwortet, einen „Deskriptivsten“ bezüglich Frage (1), jemanden, der Frage (2) mit „Nein“ beantwortet, einen „Deskriptivsten“ bezüglich Frage (2), und entsprechend für Frage (3) und (4).

\section{Relativismus}

Relativisten beantworten die erste Frage mit „Nein“: Sie bestreiten die Existenz von objektiv gültigen Prinzipien der Theoriewahl. Sie meinen zum Beispiel, dass die Prinzipien nur für die jeweilige Zeit und wissenschaftliche Disziplin gelten und nur in dem Sinn, dass sie von der jeweiligen Gemeinschaft für gültig gehalten werden. So hat Thomas Kuhn manchmal die Existenz von paradigma-unabhängigen Standards der rationalen Theoriewahl bestritten. ${ }^{3}$ Der Relativismus sucht vor allem Nachbardisziplinen der Wissenschaftstheorie wie die Wissenschaftssoziologie und die Wissenschaftsgeschichte heim. Eindeutiger als Kuhn haben sich z.B. die Vertreter der „Edinburgher Schule“ zum Relativismus bekannt. So schreiben Barry Barnes und David Bloor: „For the relativist there is no sense attached to the idea that some standards or beliefs are really rational as distinct from merely locally accepted as such. Because he thinks that there are no context-free or super-cultural norms of rationality he does not see rationally and irrationally held beliefs as making up two distinct and qualitatively different classes of thing." (1982, S. 62)

Wenn man meint, dass es keine objektiv gültigen Methoden der rationalen Theoriewahl gibt, dann hat man nichts in der Hand, womit man die Akzeptanz und Ablehnung von Theorien durch Wissenschaftler bewerten und kritisieren könnte. Dann scheint man die dritte Frage negativ beantworten zu müssen: Wir sollten uns darauf beschränken, das Verhalten der Wissenschaftler zu beschreiben, ähnlich wie Ethnologen die Normen und Gebräuche fremder Völker lediglich beschreiben und nicht bewerten sollten. ${ }^{4}$ Diese Forderung scheint in der Wissenschaftssoziologie und der Wissenschaftsgeschichte größtenteils befolgt zu werden; eine rein deskriptive Einstellung zur Wissenschaft ist dort weit verbreitet. Somit hat die zweite Frage (,Bewerten und kritisieren Wissenschaftstheoretiker die Wissenschaft, oder halten sie sich mit methodologischer Kritik zurück?"), wenn man sie für Wissenschaftssoziologen und Wissenschaftshistoriker statt Wissenschaftstheoretiker stellt, ebenfalls eine negative Antwort.

Dies ist nicht der Ort, um die Argumente der Relativisten ausführlich zu diskutieren. Wir beschränken uns daher auf einige kurze Bemerkungen, warum wir, wie die meisten

\footnotetext{
${ }^{3}$ „When paradigms enter, as they must, into a debate about paradigm choice, their role is necessarily circular. Each group uses its own paradigm to argue in that paradigm's defence.” (Kuhn 1970, S. 94)

${ }^{4}$ Zum Beispiel Barnes: „Sociology is a subject with a naturalistic, rather than a prescriptive or normative orientation; it simply tries to understand the convictions and the concepts of different cultures as empirical phenomena. External evaluation of the convictions and concepts is irrelevant to this naturalistic concern." (1982, S. 5)
} 
Wissenschaftstheoretiker, den Relativismus für sehr unplausibel halten. Wenn es keine objektiven Methoden der Theoriewahl gibt, dann stehen alle wissenschaftlichen Theorien gleich gut da, dann darf man die Wissenschaft nicht als Quelle des Wissens über die Welt akzeptieren. Dann sieht auch der praktische Erfolg der Wissenschaft wie ein Wunder aus, d.h. Relativisten können den Erfolg der Wissenschaft nicht erklären. Wissenschaftstheoretiker glauben, dass die Wissenschaftler irgendetwas richtig machen, wenn sie gewisse Theorien akzeptieren und andere zurückweisen, und wollen herausfinden, was die Wissenschaftler richtig machen. Die Theoriewahl der Wissenschaftler kann nicht nur das Ergebnis von sozialen Faktoren oder Willkür sein, das würde den Erfolg der Wissenschaft unverständlich machen. In der Wissenschaftstheorie wird somit der Relativismus nur selten vertreten; deshalb sehen wir im Folgenden von ihm ab. ${ }^{5}$

\section{Gründe für Zurückhaltung}

Betrachten wir die zweite Frage nach dem faktischen Verhalten der Wissenschaftstheoretiker, ob sie die Wissenschaft methodologisch bewerten und kritisieren oder sich aus der Wissenschaft heraushalten.

Die zweite Frage besteht aus zwei Teilen, denn sie fragt erstens, ob Wissenschaftstheoretiker die Wissenschaft bewerten und zweitens, ob sie sie kritisieren. Zur ersten Frage ist klar, dass Wissenschaftstheoretiker die Wissenschaft oft bewundern und sie für sehr erfolgreich halten (in welchem Sinn von Erfolg auch immer). So haben etwa die logischen Empiristen wie Carnap und Hempel die Wissenschaft bewundert und sie für ein Vorbild an Rationalität gehalten. Wenn man diese Einschätzung der Wissenschaft hat, dann stellt das offensichtlich eine Bewertung der Wissenschaft dar.

Diese Einstellung zur Wissenschaft ist allerdings noch nicht besonders interessant. Auch andere Wissenschaftler können ihren jeweiligen Untersuchungsgegenstand bewundern. Biologen bewundern das Wunder des Lebens, und Astronomen bewundern die Schönheit von Galaxien. Interessanter ist die Frage, ob sich Wissenschaftstheoretiker mit methodologischer Kritik in die Wissenschaften einzumischen versuchen. Zunächst könnte man meinen, dass ein Wissenschaftstheoretiker, der glaubt, es gebe objektive Methoden der rationalen Theoriewahl und er habe in seinen methodologischen Überlegungen etwas über diese Methoden herausgefunden, die Ergebnisse seiner Überlegungen bei passender Gelegenheit heranziehen wird, um das konkrete Verhalten von Wissenschaftlern einzuschätzen und gegebenenfalls zu kritisieren. Wenn ein Wissenschaftstheoretiker die richtigen Methoden der rationalen Theoriewahl oder zumindest gewisse wichtige Eigenschaften dieser Methoden herausgefunden zu haben meint, was sollte ihn dann von Kritik abhalten?

Es gibt drei Gründe, weswegen sich Wissenschaftstheoretiker mit Kritik zurückhalten. Erstens meinen die meisten Wissenschaftstheoretiker, dass die Wissenschaften, insbesondere die Naturwissenschaften, keine Kritik nötig haben, gerade weil sie schon ganz ausgezeichnet funktionieren. Verbesserungsvorschläge durch die Methodologie sind nicht erforderlich. ${ }^{6}$ Imre Lakatos verglich die Wissenschaftler mit Fischen: Wie Fische ausgezeichnet schwimmen können, ohne zu wissen, wie sie das machen, so können Wissenschaftler ausgezeichnet Wissenschaft machen, ohne zu wissen, wie sie das genau machen (Lakatos 1970, p. 148). Die Methodologie hat dann immer noch die Aufgabe herauszufinden, welches die Methoden genau sind, die die Wissenschaft so erfolgreich macht, ähnlich wie die Aufgabe der Hydrodynamik ist herauszufinden, warum die Fische so gut schwimmen können, jedoch haben weder Wissenschaftler noch Fische Kritik und Ratschläge nötig.

\footnotetext{
${ }^{5}$ Zum Relativismus siehe auch den Beitrag von Bernward Gesang in diesem Band.

${ }^{6}$ Z.B. William Newton-Smith (1981, S. 209)
} 
Der zweite Grund ist eine Folge der Hinwendung zur Wissenschaftspraxis. Kuhn, Paul Feyerabend und andere zeigten mit historischen Fallstudien, dass die von den logischen Empiristen vorgeschlagenen Methodologien die akzeptierte wissenschaftliche Praxis nicht korrekt beschreiben. Erfolgreiche Wissenschaftler verhalten sich oft nicht so, wie es die Methodologien von Carl G. Hempel, Rudolf Carnap, Karl Popper, usw. vorschreiben. Wie soll man auf den Konflikt zwischen erfolgreichem wissenschaftlichen Verhalten und von Philosophen vorgeschlagenen Methodologien reagieren? Den Wissenschaftlern die Schuld zu geben, halten viele Wissenschaftstheoretiker für anmaßend. Stattdessen gehen sie von der Annahme aus, dass bei einem Konflikt das Problem zunächst bei der jeweils vorgeschlagenen Methodologie zu suchen ist. Das aber bedeutet, dass die Wissenschaftspraxis und Wissenschaftsgeschichte als Datenquelle herangezogen wird, um Vorschläge für Methodologien zu prüfen. Wenn aber das Verhalten der Wissenschaftler als Datenbasis dient, um eine Methodologie zu prüfen, wie kann dann umgekehrt das Verhalten der Wissenschaftler auf der Grundlage der Methodologie kritisiert werden? Das wäre zirkulär.

Der dritte Grund ist ebenfalls eine Folge der Kuhn'schen Kritik. Die Inkongruenz zwischen Wissenschaftspraxis und den Methodologien wurde allgemein als Anzeichen dafür gesehen, dass die Wissenschaftstheorie noch sehr weit von der Formulierung einer korrekten Methodologie entfernt ist. Der Optimismus der frühen Jahre verflog. ${ }^{7}$ Der Eindruck herrscht heute vor, dass das Projekt einer allgemeinen Methodologie sehr viel schwieriger ist als ursprünglich angenommen. ${ }^{8}$ Solange aber noch keine allgemein akzeptierte und begründete Methodologie vorliegt, sollte man sich mit Bewertung und Kritik von Wissenschaft besser zurückzuhalten. ${ }^{9}$

Diese drei Gründe (die offensichtlich miteinander zusammenhängen) haben zur Folge, dass viele methodologische Diskussionen ausschließlich zwischen Wissenschaftstheoretikern ablaufen. In den wissenschaftstheoretischen Diskussionen, z.B. in den Auseinandersetzungen um den Bayesianismus, kritisieren sich die Wissenschaftstheoretiker nur gegenseitig und nicht die Einzelwissenschaften. In weiten Teilen der Wissenschaftstheorie dominiert daher ein de-facto-Deskriptivismus. Beliebt ist der Vergleich der Wissenschaftstheorie mit der Astronomie, welche auch nur daran interessiert ist, Sterne und Galaxien zu beobachtet und zu beschreiben, und nicht, sie irgendwie zu verändern oder zu verbessern. ${ }^{10}$

\section{Kritik an Wissenschaft}

Zwar sind viele methodologische Diskussionen nur an die Kollegen innerhalb der Wissenschaftstheorie gerichtet, aber das gilt nicht für alle methodologischen Diskussionen. Die Wissenschaft funktioniert nicht immer so gut, wie von Vertretern des ersten Grundes behauptet. Wenn Wissenschaftstheoretiker meinten, dass Wissenschaft schlecht funktioniert, dann haben sie ihre Zurückhaltung oft aufgeben und Wissenschaft durchaus kritisiert. So hat Popper bekanntlich die Psychoanalyse, den Marxismus und die Astrologie kritisiert und ihnen die Wissenschaftlichkeit gleich ganz aberkannt. Dabei ging er von seiner eigenen Methodologie aus.

\footnotetext{
${ }^{7}$ Hier ist ein Beispiel für die Einstellung der frühen Jahre: "Hier ist noch vieles umstritten. Ich bin aber sehr optimistisch und glaube, dass wir auch zahlenmäßig die Wahrscheinlichkeit angeben können, wenn etwa auf der einen Seite gewisse Daten gegeben sind und auf der anderen Seite eine Hypothese, die sich auf Unbekanntes bezieht." (Carnap 1993, S. 143)

${ }^{8}$ Wie schwierig das Projekt ist, zeigt z.B. die Debatte um den wissenschaftlichen Realismus.

${ }^{9}$ Später werden wir nach den möglichen Gründen für den Mangel an Erfolg in der Methodologie fragen.

${ }^{10}$ Z.B. Peter Lipton (2004). Es gibt noch einen vierten Grund für die Zurückhaltung der Wissenschaftstheoretiker, den wir hier allerdings nicht weiter diskutieren werden: Viele der vorgeschlagenen Methodologien sind sehr abstrakt und formal, was ihren Einsatz zur Bewertung von konkreter wissenschaftlicher Praxis erschwert und oft unmöglich macht.
} 
Auch die beiden anderen Gründe für die Zurückhaltung der Wissenschaftstheoretiker gelten nur mit Einschränkungen. So war die Argumentation, die wir eben für den zweiten Grund gegeben haben, zu einfach. Selbst wenn der Methodologe für die Begründung von Prinzipien der Theoriewahl keine andere Datenbasis als die Wissenschaftspraxis zugrunde legen will, wird er nur diejenigen Teile der Wissenschaft als Datenbasis verwenden, von denen er meint, dass sie gut funktionieren, d.h. die er für erfolgreich oder vorbildlich hält. Wenn er aus erfolgreicher Wissenschaft Methoden herausliest, dann kann er die herausgelesenen Methoden dazu benutzen, andere Teile der Wissenschaftspraxis zu kritisieren, die er für weniger vorbildlich hält. Zum Beispiel mag ein Methodologe aus der Wissenschaftspraxis ableiten, dass zuverlässige Aussagen über die Wirkungen von Medikamenten nur gemacht werden können, wenn die Medikamente mit Doppelblindversuchen getestet wurden. Mit dieser methodologischen Einsicht könnte der Methodologe dann Fälle kritisieren, in denen Aussagen über Medikamente gemacht werden, die nicht das Ergebnis von Doppelblindversuchen sind.

Auch der dritte Grund, das Fehlen einer allgemein akzeptierten Methodologie, führt nicht zum Ausschluss jeglicher Kritik. Wissenschaft kann kritisiert werden, wenn das jeweilige wissenschaftliche Fehlverhalten so gravierend ist, dass keine bestimmte Methodologie zugrunde gelegt werden muss, um die methodologischen Fehler einzusehen. Dann kann die Wissenschaftstheorie methodologische Kritik an der Wissenschaft üben, obwohl noch keine allgemein akzeptierte Methodologie vorliegt. So gibt es heute eine ganze Reihe von Beispielen von Kritik an schlechter Wissenschaft und Pseudowissenschaft. Zum Beispiel tadelt Kitcher die Soziobiologie für die ungenügenden empirischen Begründungen ihrer Hypothesen. ${ }^{11}$ Patricia Kitcher (1992) und Adolf Grünbaum (1988) werfen Freud vor, empirische Evidenzen ignoriert und den Anschluss an benachbarte empirische Disziplinen verloren zu haben. Clark Glymour kritisiert prominente Arbeiten der Intelligenzforschung: Sie gelangten zu Schlussfolgerungen, die nicht durch die Daten belegt seien. In den so genannten „science wars" beklagen Wissenschaftler und Philosophen den Verfall wissenschaftlicher Standards in den Literaturwissenschaften und verwandten Geisteswissenschaften (Alan Sokal 2001, Brown 2001, Ian Hacking 1999). Und so weiter. In all diesen Fällen nehmen Wissenschaftstheoretiker konkretes wissenschaftliches Verhalten (oder was sich dafür hält) unter die Lupe und kritisieren es. Es gibt also viele Fälle, in denen sich die Wissenschaftstheoretiker mit präskriptiver Absicht in die Wissenschaften einmischen und die Wissenschaft verbessern wollen. Der Vergleich der Wissenschaftstheoretiker mit Astronomen gilt somit nur begrenzt; Wissenschaftstheoretiker haben durchaus die Bereitschaft, Wissenschaft zu kritisieren. Folglich hat ein Deskriptivist bezüglich Frage (2) Unrecht. ${ }^{12}$

\section{Dürfen die das?}

Wenden wir uns der dritten Frage zu: Sind Wissenschaftstheoretiker dazu berechtigt, die Wissenschaft zu bewerten und zu kritisieren? Der Deskriptivist bezüglich Frage (2) meint „Nein“ und fordert, dass sich Wissenschaftstheoretiker aus der Wissenschaft heraushalten.

\footnotetext{
${ }^{11}$ Kitcher (2003, Kapitel 11 bis 16)

${ }^{12}$ Es ist sicherlich auch eine Sache des Temperaments des einzelnen Wissenschaftstheoretikers, wie stark er sich in die Wissenschaft einmischt. Kuhn und Feyerabend unterschieden sich deutlich in ihrer Neigung, die Wissenschaft zu kritisieren. In Kuhns Schriften ist oft schwer zu erkennen, ob er seine Behauptungen normativ oder deskriptiv meint (Peter GodfreySmith 2003, S. 79), während Feyerabend lautstark Forderungen erhob, wie Wissenschaft vorzugehen habe. Auch Popper litt nicht eben unter einem Mangel an Selbstwertgefühl und mischte sich kräftig in die Wissenschaft ein, wohingegen Carnap philosophisch sehr tolerant war und unfähig, Personen und Leistungen zu kritisieren, die er bewunderte.
} 
Doch überzeugt uns das nicht. Abgesehen davon, dass der Deskriptivist hier selbst macht, was er den Wissenschaftstheoretikern nicht zugestehen will, nämlich anderen Leuten Vorschriften zu machen, ist nicht zu sehen, aus welchen Gründen man den Wissenschaftstheoretikern die Bewertung und Kritik von Wissenschaft und Wissenschaftlern verbieten soll. Kritik und Gegenkritik ist ein wesentliches Element von Wissenschaft, ohne sie hätte sie kaum den großen Erfolg, den sie heute hat: Warum sollen sich Wissenschaftstheoretiker nicht daran beteiligen dürfen? Im Prinzip sollte sich jeder daran beteiligen dürfen, der die nötige Kompetenz besitzt. Die Methoden der Wissenschaft, ihre Korrektheit und Inkorrektheit, wann folglich Kritik und Gegenkritik in der Wissenschaft zutreffend sind, das sind gerade die Themen der Methodologie, da sollte man hoffen, dass die Wissenschaftstheoretiker ein gewisses Maß an Kompetenz erworben haben.

Zwar haben wir eben drei Gründe gesehen, warum es für den Methodologen oft klug ist, Zurückhaltung zu üben, aber wir haben auch bemerkt, dass diese drei Gründe nur eingeschränkt gelten. Insbesondere was den dritten Grund angeht, sollte man hoffen, dass die Arbeit der Methodologen nicht völlig ohne Ergebnisse geblieben ist, selbst wenn sie noch keine allgemeingültige Methodologie vorlegen können. Wenn das der Fall ist, warum sollen sie ihr Fachwissen dann nicht anwenden dürfen, und das bedeutet, konkretes wissenschaftliches Verhalten mit Hilfe der Ergebnisse der Methodologie einzuschätzen und gegebenenfalls zu kritisieren. Die obigen Beispiele der Soziobiologie, der Intelligenzforschung, der ,science wars“, usw. sind denn auch plausible Beispiele dafür, wie Wissenschaftstheoretiker einen nützlichen Beitrag zur Wissenschaft liefern können. Dass die Wissenschaftstheoretiker die Wissenschaft bewerten und kritisieren, kann man also nicht beanstanden.

Bezüglich der dritten Frage unterscheidet sich die Wissenschaftstheorie nicht von anderen Wissenschaften. ${ }^{13}$ Der Frage, ob die Wissenschaftstheoretiker ihr Wissen anwenden und sich in die Wissenschaft einmischen dürfen, entspricht die Frage, ob Ökonomen, Psychologen oder Statistiker ihr entsprechendes Wissen anwenden dürfen und sich in die entsprechenden Bereiche einmischen dürfen. In allen Fällen gibt es keinen Grund, weswegen man eine Einmischung verbieten soll, wenn die entsprechende Kompetenz vorliegt. Wenn Ökonomen Fachwissen über wirtschaftliche Zusammenhänge besitzen, warum sollen sie sich dann nicht beispielsweise in die Wirtschaftspolitik einmischen dürfen.

\section{Aufgaben und Pflichten der Wissenschaftstheoretiker}

Betrachten wir schließlich die letzte Frage, ob Wissenschaftstheoretiker die Pflicht haben, sich in die Wissenschaft einzumischen. Diese Frage lässt zwei Lesarten zu. Zum einen kann sie fragen, ob es zu den Aufgaben der Wissenschaftstheoretiker gehört, die Wissenschaft methodologisch zu kritisieren, zum zweiten kann sie fragen, ob Wissenschaftstheoretiker in irgendeinem anderen Sinn zur kritischen Begleitung oder Kommentierung von Wissenschaft verpflichtet sind. ${ }^{14}$

Was die Aufgaben von Wissenschaftstheoretikern angeht, so scheint uns, dass ihre eigentliche Aufgabe der Erwerb von Wissen ist. Auch hier unterscheidet sich der Wissenschaftstheoretiker nicht von anderen Wissenschaftlern. Auch von einem Psychologen als Wissenschaftler erwartet man nur, dass er etwas über das menschliche Verhalten, Denken, Fühlen, usw. herausfindet, nicht aber, dass er sich in die therapeutische Praxis einmischt. Ebenso wenig erwartet man von einem Bauingenieur als Wissenschaftler, dass er Brücken

\footnotetext{
${ }^{13}$ Vergleiche auch Spohn (2003).

${ }^{14}$ Es gibt natürlich eine große Debatte um die Verantwortung von Wissenschaftlern, doch dreht sich diese Debatte vornehmlich um den Gebrauch und Missbrauch von konkretem wissenschaftlichem Wissen.
} 
und Straßen bewertet und kritisiert. Das steht nicht in seiner Stellenbeschreibung, wenn er an einer Universität oder anderen wissenschaftlichen Einrichtung angestellt ist. Wenn die Ausgangsfrage, ob die Wissenschaftstheorie eine normative Disziplin ist, bedeuten soll, dass es zu den Aufgaben der Wissenschaftstheorie gehört, sich in die Wissenschaft einzumischen, dann ist sie keine normative Disziplin, aber dann ist fraglich, ob es überhaupt eine wissenschaftliche Disziplin gibt, die in diesem Sinn eine normative Disziplin ist.

Es gibt noch eine zweite Lesart der vierten Frage. Selbst wenn der Wissenschaftstheoretiker nicht die Aufgabe hat, sich in die Wissenschaft einzumischen, hat er vielleicht auf Grund seiner Kompetenz die moralische Pflicht, die Wissenschaft kritisch zu begleiten und sich mitunter einzumischen. So meint Popper: "Everyone has a special responsibility in the field in which he has either special power or special knowledge" (1994, S. 128). Wenn es in der Wissenschaft z.B. Fehlentwicklungen gibt, die der Wissenschaftstheoretiker auf Grund seiner besonderen Ausbildung oder seines Fachwissens aufdecken kann, dann sollte er das tun. Das meint zumindest Noretta Koertge bezüglich der oben erwähnten ,science wars": ,'New age' critiques of science are figuring in decisions about making agricultural technology and medicine available to developing countries (...) and regulations affecting genetic research and the archaeologist's access to fossils. If we have reason to believe these philosophies are unsound and are leading to bad social results, then it is our moral responsibility to speak out." (2000, S. 679) Wir sympathisieren mit Koertges Forderung, doch sind wir damit bei sehr großen und tiefen Problemen angelangt, nämlich Problemen der moralischen Verantwortung, die wir in diesem Aufsatz nicht angemessen behandeln können.

\section{Methodologie im weiten Sinne}

Damit haben wir die vier Versionen der Eingangsfragen beantwortet - allerdings nur für die Methodologie, verstanden in dem engen Sinn unserer obigen Definition. In der Wissenschaftstheorie gibt es Probleme, die in einem weiten Sinn methodologisch sind. Ihre jeweilige Form von Normativität müsste ebenfalls ausführlich diskutiert werden, was wir an dieser Stelle jedoch nicht machen können. Wir beschränken uns darauf, sie kurz zu erwähnen. $\mathrm{Da}$ ist die feministische Wissenschaftskritik, etwa die Kritik an der Primatenforschung, wonach das Geschlecht des Forschers eine Rolle bei der Entwicklung und Akzeptanz von Hypothesen spielte (Sarah Blaffer Hredy 2002). Dann haben wir das das Problem der Interpretation von wissenschaftlichen Theorien wie der Quantenmechanik oder der statistischen Mechanik, auf das Lawrence Sklar jüngst hingewiesen hat. Das Bemerkenswerte ist dabei für Sklar, dass der Anstoß für diese Untersuchungen aus den Einzelwissenschaften selbst kommt: "[T]he essential motivation for interpretation comes from within science itself. Demands for the interpretation or reinterpretation of a theory come from a sense, within the theoretical scientific community itself, that 'something has gone wrong' with the theory itself." 15

Drittens haben wir die Frage, ob es auch Normen für den so genannten Kontext der Entdeckung gibt. So wollen Clark Glymour et. al. (1993) die Bayes'schen Netze für die Entdeckung von kausalen Hypothesen einsetzen. Viertens gibt es die Debatte um den wissenschaftlichen Realismus und van Fraassens Antirealismus. Und so weiter. Eine vollständige Diskussion der unterschiedlichen Formen von Normativität, mit denen sich die Wissenschaftstheorie beschäftigt, müsste all diese Themen aufgreifen, was jedoch den Rahmen dieses Aufsatzes sprengen würde.

\footnotetext{
${ }^{15}$ Sklar 2000, S. 730; siehe auch Lipton 2004.
} 


\section{Das Elend der Methodologie}

Wie wir gesehen haben, ist eines der Motive, weswegen sich Wissenschaftstheoretiker mit Kritik an konkretem wissenschaftlichen Verhalten zurückhalten, dass die Methodologie noch nicht besonders weit entwickelt zu sein scheint. Diese Situation wollen wir nun genauer untersuchen.

Wenn man die Errungenschaften der Wissenschaft mit den Errungenschaften der Methodologie vergleicht, dann fällt in der Tat auf, dass es hier eine sehr große Diskrepanz gibt. Auf der einen Seite hat die Wissenschaft in den letzten 300 Jahren gewaltige Fortschritte gemacht, die Vorhersagen und Einflussnahme in der Welt in einem Ausmaß ermöglichen, von der frühere Generationen nur träumen konnten. Auf der anderen Seite haben wir die Methodologie. Sie soll die Prinzipien der rationalen Theoriewahl herausfinden und begründen, auf denen die gewaltigen Erfolge der Wissenschaft beruhen. Doch scheint sie in einem bedauernswerten Zustand zu sein. Obwohl sich Wissenschaftler und Philosophen seit Hunderten von Jahren mit der Methodologie beschäftigen, sind sie weit davon entfernt, einen Methodenkanon zu formulieren und zu begründen, der allgemein akzeptiert werden könnte.

Die Diskussion in der Methodologie hat alle Eigenschaften, die für philosophische Diskussionen typisch sind: Es gibt verschiedene Positionen und verschiedene Argumente zur Stützung und Entkräftung der verschiedenen Positionen, doch gibt es keine Entscheidung und ein Ende der Meinungsverschiedenheiten ist nicht in Sicht. Warum ist die Diskrepanz zwischen Wissenschaft und Wissenschaftstheorie so groß? Warum sind die Methodologen so erfolglos, während die Methoden selbst so erfolgreich sind? Warum ist die Methodologie immer noch eine philosophische Disziplin? Wir wollen vier Möglichkeiten betrachten, wie man die Probleme der Methodologie erklären kann.

Viele Wissenschaftstheoretiker meinen, dass es eine extrem schwierige Aufgabe ist, die wissenschaftlichen Methoden zu beschreiben und zu begründen. Eine Erklärung, die hierfür angegeben wird, ist, dass die wissenschaftlichen Methoden tief in der wissenschaftlichen Praxis verborgen liegen, was eine systematische Beschreibung sehr schwer macht. Und solange keine systematische Beschreibung der wissenschaftlichen Methoden vorliegt, können sie auch nicht allgemein begründet werden. Diese Erklärung des bisherigen Misserfolgs der Methodologie sieht das Verhältnis zwischen der wissenschaftlichen Praxis und der Beschreibung dieser Praxis als ein Verhältnis zwischen „knowing-how“ und „knowing-that“. Erinnern wir uns an Lakatos' Vergleich von Fischen mit Wissenschaftlern: Genau wie Fische die Fähigkeit haben, anstrengungslos und effizient zu schwimmen, ohne die Hydrodynamik zu kennen, können Wissenschaftler etwas über die Welt herausfinden, ohne zu wissen, was für Methoden sie dabei anwenden und warum die Methoden erfolgreich sind.

Doch gibt es hier mindestens eine Disanalogie. Die Gleichungen der Hydrodynamik sind extrem kompliziert, weswegen eine präzise Beschreibung der Schwimmfähigkeit von Fischen nicht möglich ist. Stattdessen müssen die Physiker zu Näherungsmethoden und idealisierenden Modellen greifen. Jedoch sind sich die Physiker darüber einig, was die grundlegenden Gleichungen sind, die für die Strömungen im Wasser gelten, und es ist klar, dass die Modelle besser werden, wenn die Rechenleistung der Computer steigt. Diese Art von Fortschritt können wir in der Methodologie nicht beobachten. Im Gegenteil, wenn man sich die von Philosophen vorgeschlagenen Methodologien anschaut, dann fällt auf, dass sie sehr einfach sind. Poppers Falsifikationismus lässt sich in wenigen Sätzen erläutern. Das gleiche gilt für die hypothetisch-deduktive Methode, die von W. V. Quine und Hempel vertreten wurde. Der Bayesianismus ist etwas komplizierter, aber seine zentralen Behauptungen lassen sich, wie wir gleich feststellen werden, jedem, der mit den vier Grundrechenarten vertraut ist, in höchstens ein paar Stunden erklären. Der Misserfolg der Methodologen liegt also sicher nicht daran, dass die Prinzipien der Theoriewahl besonders kompliziert sind. 


\section{Relativismus und Unexplizierbarkeit}

Weil also ein Fortschritt und eine Einigung in der Methodologie nicht in Sicht zu sein scheinen, wurden radikalere Möglichkeiten zur Erklärung des Misserfolgs der Methodologie vorgeschlagen. Drei radikale Erklärungen möchten wir ansprechen. Die erste wird von manchen Relativisten insbesondere Wissenschaftssoziologen vertreten. Sie meinen, die Theoriewahl der Wissenschaftler müsse mit sozialen Faktoren, wie Interessen, Machtverhältnissen, usw. erklärt werden, statt mit objektiven paradigma-übergreifenden Regeln der Theoriewahl, die es nicht gebe. Wie oben bemerkt, halten Philosophen diese Ansätze für sehr unplausibel. Wenn die Theoriewahl der Wissenschaftler nur durch soziale Faktoren und Kräfte bestimmt ist, dann kann nicht erklärt werden, warum sich mit Hilfe von wissenschaftlichen Theorien Flugzeuge und Radios bauen lassen.

Eine zweite Möglichkeit, wie der Misserfolg der Methodologen erklärt werden könnte, ist die Behauptung, dass Wissenschaftler zwar in der Lage sind, vernünftig zwischen verschiedenen Theorien zu entscheiden, dass aber ihre Fähigkeit zur vernünftigen Entscheidung prinzipiell nicht explizierbar ist. So behauptet Kuhn, es gebe keine mechanischen Algorithmen für Theoriewahl. Polanyi spricht von „tacit rationality“ und von einem ,tacit incommunicable feel for the right thing”. Duhem nennt diese Fähigkeit "good sense", den gute Wissenschaftler besitzen, wenn sie zwischen Theorien entscheiden. Brown, Howard Sankey, Newton-Smith u.a., sprechen vom „Urteilsvermögen“ (,jugdement“) von Wissenschaftlern, welches nicht expliziert werden kann. Die Behauptung ist also, dass es prinzipielle Grenzen dafür gibt, was die Wissenschaftstheorie explizieren kann, und dass das Urteilsvermögen der Wissenschaftler bei der Wahl zwischen konkurrierenden Theorien jenseits der Grenze des Explizierbaren liegt. ${ }^{16}$

Doch auch diese Erklärung ist unbefriedigend. Betrachten wir einen Wissenschaftler, der zwischen zwei Theorien wählt und sich dabei auf sein Urteilsvermögen verlässt, welches angeblich unexplizierbar ist. Nehmen wir an, seine Wahl ist in irgendeinem Sinn vernünftig. Wenn das der Fall ist, dann müssen die Theorien gewisse Eigenschaften - nennen wir sie „gutmachende“ Eigenschaften - besitzen, die seine Wahl zu einer vernünftigen Wahl machen und die irgendwie vom Wissenschaftler erfasst werden müssen. Auch wenn der Wissenschaftler selbst nicht sagen kann, welche Eigenschaften das sind, so müssen sie doch in irgendeiner Weise in seinem kognitiven Apparat repräsentiert oder implementiert sein. Dann stellt sich die Frage, warum es prinzipiell unmöglich sein soll, sowohl die gutmachenden Eigenschaften wie ihre Repräsentation im kognitiven Apparat des Wissenschaftlers zu beschreiben. Wenn der kognitive Apparat des Wissenschaftlers die gutmachenden Eigenschaft repräsentieren kann, warum sollen wir sie dann nicht auch explizit beschreiben können? Aus welchem Grund sollen unsere sprachlichen Mittel an dieser Aufgabe scheitern?

\section{Partikularismus}

\footnotetext{
${ }^{16}$ Newton-Smith (1981, Kapitel 8), Brown (1988), Sankey (1997, Kapitel 8), Larry Laudan (1996, Kap. 1.3). Pierre Duhem zitiert Blaise Pascal: "The heart has its reason which reason knows nothing of." (Siehe Donald Gillies 1998, S. 310). Diese Behauptung wird selten in dieser Stärke vertreten, ist jedoch in abgeschwächter Form überraschend weit verbreitet. Die meisten Philosophen, die sie in abgeschwächter Form vertreten, versuchen dennoch, explizite Regeln der Theoriewahl zu formulieren. Sie glauben dann nicht mehr, dass wir eine vollständige Charakterisierung der Regeln der Theoriewahl geben können, sondern nur eine Teilcharakterisierung.
} 
Der Partikularismus präsentiert eine dritte Erklärung für die Diskrepanz zwischen den Leistungen der Wissenschaft und den Leistungen der Methodologie. Er bestreitet nicht die Möglichkeit einer Beschreibung der gutmachenden Eigenschaften von Theorien, doch bestreitet er die Möglichkeit einer systematischen und einfachen Beschreibung. Eine solche Beschreibung könne es nicht geben, denn die gutmachenden Eigenschaften und die Methoden der rationalen Theoriewahl seien extrem vielfältig und unterschieden sich von Kontext zu Kontext. ${ }^{17}$ Die Vielfalt und Kontextabhängigkeit erkläre zum einen, warum es in der Methodologie fortgesetzten Dissens und keine Erfolge gebe, und zum anderen, warum das Urteilsvermögen der Wissenschaftler so schwer zu explizieren sei.

Der Partikularismus leugnet nicht, dass es einige allgemeine Regeln für Theoriewahl gibt, doch behauptet er, dass es sich dabei nur um sehr einfache und unkontroverse Faustregeln handelt, wie die Regeln, dass Theorien der Empirie nicht widersprechen dürfen und dass sie konsistent sein sollten. Und selbst diese Regeln gelten womöglich nur eingeschränkt. So hat Feyerabend die Akzeptanz von widersprüchlichen Theorien verteidigt, etwa im Falle von Bohrs Atommodell, und Kuhn, Lakatos und andere waren sich einig, dass praktisch jede Theorie gewisse Probleme mit der Empirie hat.

Der Partikularismus unterscheidet sich vom Relativismus. Beim Partikularismus kann die Korrektheit oder Gültigkeit eine Methode vom jeweiligen Gegenstandsbereich und vom Wissenstand der Wissenschaftler abhängen. Aber wenn eine Methode für einen gewissen Gegenstandsbereich und für einen gewissen Wissensstand gültig ist, dann ist sie für alle Gruppen und Kulturen mit diesem Wissensstand, die etwas über diesen Gegenstandsbereich herausfinden wollen, gültig. Dagegen hängt beim Relativismus die Gültigkeit von der jeweiligen Kultur oder wissenschaftlichen Gemeinschaft ab; über die Akzeptanz der Methode in der jeweiligen Kultur hinaus, gibt es gar keinen Sinn von Gültigkeit oder Korrektheit von Methoden. Daher sind die beiden Arten von Abhängigkeit grundverschieden. ${ }^{18}$

Alle methodologischen Prinzipien, die über die Faustregeln hinausgehen, haben also nur lokale Gültigkeit und gelten nur für bestimmte historische Epochen und bestimmte wissenschaftliche Disziplinen oder Subdisziplinen. Das wollen wir am Beispiel eines wichtigen Bewertungskriteriums für Theorien, dem Kriterium der Einfachheit, erläutern. Dabei beschränken wir uns auf das Problem, Einfachheit zu charakterisieren, und sagen nichts zum Problem, ob Einfachheit ein Wahrheitsindikator ist.

Philosophen haben bisher vergeblich versucht, eine allgemeine Charakterisierung von Einfachheit zu finden. Die vorliegenden Versuche (insbesondere die formalen) sehen nicht besonders attraktiv aus, und es scheint wenige Philosophen zu geben, die so etwas noch versuchen. Eine Theorie kann auf viele verschiedene Weisen einfach sein. Erstens kann die Zahl der Prozesse oder Entitäten niedrig sein. Das Problem ist dann, dass Prozesse und Entitäten auf verschiedene Weisen gezählt werden können. Ist der Atomismus der modernen Chemie kompliziert, weil er 92 verschiedene Elemente annimmt, oder einfach, weil es für ihn nur eine Sorte von Entitäten gibt, nämlich Atome (Kosso, 1992, S. 45)? Statt der Prozesse und Entitäten kann man zweitens die Grundannahmen oder Axiome der Theorie zählen. Aber für jede Theorie gibt es viele verschiedene Formulierungen, für die die Zahl von Grundannahmen sehr verschieden sein kann. Drittens kann eine Theorie einfach sein, weil ihre Gleichungen

\footnotetext{
${ }^{17}$ Einen Einstieg in die Literatur des Partikularismus bieten zum Beispiel Nola/Sankey (2000, S. 26-36) und die Beiträge in Galison/Stump (1996).

${ }^{18}$ Eine andere Erläuterung des Unterschiedes zwischen Partikularismus und Relativismus geben Laudan (1984) und Sankey (1997). Sie gehen von der Annahme aus, dass Methoden dazu dienen, epistemische Ziele zu erreichen. Es ist dann eine objektive nicht-relative Frage, ob eine Methode dazu taugt, ein gegebenes epistemisches Ziel zu erreichen. Die Objektivität der Methoden ist verträglich mit der Variabilität der Methoden, und die Variabilität der Methoden führt nicht automatisch zu einem Relativismus. (Bei Laudan variieren auch die epistemischen Ziele, während Sankey Wahrheit oder Wahrheitsnähe als ein unveränderliches Ziel der Wissenschaft annimmt.) Unseres Erachtens ist jedoch die Annahme einer instrumentellen Natur von Methoden, so plausibel sie ansonsten ist, nicht nötig, um Partikularismus und Relativismus voneinander zu unterscheiden.
} 
einfach sind. Doch hängt die Kompliziertheit der Gleichungen z.B. von der Wahl der Koordinaten ab. Viertens scheinen uns all diese Maßstäbe in vielen Fällen nicht viel weiter zu helfen. Wie soll man sie z.B. auf die Frage anwenden, ob die Evolutionstheorie einfacher oder komplizierter ist als der Kreationismus (Kosso, 1992, S. 44)? Der Partikularismus behauptet mithin, dass es keine allgemeine Theorie der Einfachheit gibt; Einfachheit selbst ist nichts Einfaches.

\section{Der Bayesianismus}

Die wichtigste nicht-partikularistische Methodologie ist heute der Bayesianismus, den wir nun vorstellen werden. ${ }^{19}$ Es versteht sich von selbst, dass wir nur die Grundlagen des Bayesianismus darstellen können, denn die Diskussion um den Bayesianismus hat inzwischen einen gewaltigen Umfang angenommen.

Der Bayesianismus beschreibt die Überzeugungen und Überzeugungsänderungen eines ideal rationalen Subjektes. Er nimmt an, dass die Überzeugungen eines solchen Subjekts verschiedene Stärken oder Grade haben. Jede Überzeugung des Subjekts besteht aus einer Aussage $A$ und dem Glaubensgrad $\mathrm{P}(A)$, der den Grad der Unsicherheit des Subjekts bezüglich der Aussage $A$ misst. Die Glaubensgrade sind reelle Zahlen aus dem Intervall $[0,1]$. Sie sind rational, wenn sie den Axiomen der Wahrscheinlichkeitstheorie gehorchen. Diese Axiome sind:

(1) $\mathrm{P}(A) \geq 0$, für alle Aussagen $A$,

(2) $\mathrm{P}(A)=1$, wenn $A$ eine logische oder mathematische Wahrheit ist,

(3) $\mathrm{P}(A$ oder $B)=\mathrm{P}(A)+\mathrm{P}(B)$, wenn $A$ und $B$ sich widersprechen.

Um zu zeigen, dass die Glaubensgrade eines Subjekts nur dann rational sind, wenn sie den Wahrscheinlichkeitsaxiomen gehorchen, bietet der Bayesianismus die „Dutch-BookArgumente“ an. Diese verbinden die Glaubensgrade des Subjekts mit den Präferenzen des Subjekts. Nehmen wir an, die Glaubensgrade eines Subjekts verletzten eines der drei Axiome. Dann gibt es für die zugehörigen Aussagen eine gewisse Kombination von Wetten (ein „Dutch Book“), die für das Subjekt eine unangenehme Eigenschaft hat: Obwohl das Subjekt die Wettkombinationen für fair hält, wird es in jedem Fall, egal welche der Aussagen sich als wahr herausstellt, einen Verlust machen. Wenn aber schon beim Abschluss der Wetten für das Subjekt klar ist, dass sie zu einem sicheren Verlust führen, dann können die Glaubensgrade und Präferenzen des Subjekts nicht rational sein.

Dutch Books werden allerdings nicht von allen Bayesianern als Begründungen für die Wahrscheinlichkeitsaxiome akzeptiert. Sie sind von pragmatischer Natur, weil sie eine Inkohärenz in der Präferenzstruktur des Subjekts aufdecken. Viele Philosophen würden eine epistemische Begründung der Wahrscheinlichkeitsaxiome bevorzugen, also eine Begründung, die zeigt, dass ein Subjekt auf irgendeine Weise das Ziel Wahrheit kompromittiert, wenn seine Glaubensgrade nicht den Wahrscheinlichkeitsaxiomen gehorchen. Ein Versuch in diese Richtung ist James Joyce (1998).

Für die Untersuchung der zeitlichen Änderung von rationalen Meinungen benötigen wir den Begriff der bedingten Wahrscheinlichkeit $\mathrm{P}(A \mid B)$ von $A$ unter der Bedingung $B$. Diese

\footnotetext{
${ }^{19}$ Einführungen in den Bayesianismus bieten z.B. Colin Howson und Peter Urbach (1993), Brian Skyrms (1990), John Earman (1992) und Paul Horwich (1982).
} 
sind definiert durch $\mathrm{P}(A \mid B)=\mathrm{P}(B$ und $A) / \mathrm{P}(B)$, falls $\mathrm{P}(B)>0$. Damit können wir explizieren, was es heißt, dass eine Hypothese $\mathrm{H}$ durch eine Evidenz E bestätigt oder entkräftet wird:

E stützt $\mathrm{H}$, falls $\mathrm{P}(H \mid E)>\mathrm{P}(H)$. E entkräftet $\mathrm{H}$, falls $\mathrm{P}(H \mid E)<\mathrm{P}(H)$.

Die Differenz $\mathrm{P}(H \mid E)$ - $\mathrm{P}(H)$ ist dann ein Maß für die Stärke der Bestätigung. Es gibt noch eine ganze Anzahl weiterer Maße, mit denen die Stärke der Stützung einer Hypothese durch eine Beobachtung gemessen werden kann. Branden Fitelson (1999) vergleicht diese Maße miteinander und erörtert ihre Vor- und Nachteile.

Mit der bedingten Wahrscheinlichkeit können wir eine Regel formulieren, wie das Subjekt seine Überzeugungen auf rationale Weise ändern sollte, wenn es neue Beobachtungen macht. Die Konditionalisierungsregel besagt, dass, wenn das Subjekt die Beobachtung $E$ macht, für alle Aussagen $A$ die neuen Wahrscheinlichkeiten des Subjekts die nach der Beobachtung $E$ bedingten Wahrscheinlichkeiten $\mathrm{P}(A \mid E)$ sind. Die Konditionalisierungsregel folgt nicht aus den Axiomen der Wahrscheinlichkeitstheorie, wird jedoch durch die Interpretation der Wahrscheinlichkeiten als Glaubensgrade nahe gelegt. Sie lässt sich ebenfalls durch ein Dutch-Book-Argument begründen, wie David Lewis gezeigt hat (1999, Kap. 23).

An dieser Stelle ist es üblich, darauf hinzuweisen, dass die Konditionalisierungsregel die unrealistische Annahme macht, dass das Subjekt die hereinkommende Evidenz $E$ für absolut gewiss hält, und dass Richard Jeffrey (1983) deswegen eine andere Änderungsregel vorgeschlagen hat, die so genannte Jeffrey-Konditionalisierung, die das Problem der Konditionalisierungsregel vermeidet. Wenn man sich die Literatur jedoch genauer anschaut, dann wird klar, dass die Jeffrey-Konditionalisierung praktisch nie eingesetzt wird. Alle Anwendungen des Bayesianismus verwenden nur die gewöhnliche Konditionalisierungsregel. Dass das Subjekt die Evidenz $E$ für absolut gewiss hält, wird als eine Idealisierung akzeptiert, die die Anwendungen des Bayesianismus nicht weiter beeinträchtigt.

Um die bedingten Wahrscheinlichkeiten zu berechnen, benötigen wir das Bayes'sche Theorem, das aus den Wahrscheinlichkeitsaxiomen folgt:

$$
\mathrm{P}(H \mid E)=\mathrm{P}(H) \cdot \mathrm{P}(E \mid H) / \mathrm{P}(E) .
$$

Hier heißt $\mathrm{P}(H)$ „Anfangswahrscheinlichkeit“ (englisch „prior“), $\mathrm{P}(H \mid E)$ heißt „Endwahrscheinlichkeit" (englisch „posterior") und $\mathrm{P}(E \mid H)$ heißt „Likelihood“. Mit Hilfe dieser Formel kann die Endwahrscheinlichkeit aus der Anfangswahrscheinlichkeit von $H$, der Likelihood und der Anfangswahrscheinlichkeit von $E$ berechnet werden.

\section{Vorzüge des Bayesianismus}

Das eben erwähnte Bayes'sche Theorem steht im Zentrum des Bayesianismus. Mit seiner Hilfe kann der Bayesianismus viele Intuitionen und methodologische Regeln begründen. Schon durch die bloße Betrachtung des Bayes'schen Theorems ergeben sich ein paar ganz einfache Zusammenhänge, die gut im Einklang mit unseren Intuitionen über die Bestätigungsbeziehung zwischen Beobachtung und Theorie stehen. Im Bayes'schen Theorem taucht auf der rechten Seite der Quotient $\mathrm{P}(E \mid H) / \mathrm{P}(E)$ auf. Dieser Quotient misst die Korrelation zwischen $H$ und $E$. Wenn $H$ und $E$ positiv korreliert sind, das heißt $\mathrm{P}(E \mid H)$ größer als $\mathrm{P}(E)$ ist, dann erhöht sich die Wahrscheinlichkeit der Hypothese durch das Eintreffen der Beobachtung, also $\mathrm{P}(H \mid E)>\mathrm{P}(H)$, mit anderen Worten, $H$ wird durch $E$ bestätigt. Je größer die positive Korrelation zwischen $H$ und $E$ ist, d.h. je größer $\mathrm{P}(E \mid H)$ verglichen mit $\mathrm{P}(E)$ ist, 
desto stärker wird $H$ durch $E$ bestätigt. Analog dazu wird $H$ durch $E$ entkräftet, also $\mathrm{P}(H \mid E)<$ $\mathrm{P}(H)$, wenn $\mathrm{P}(E \mid H)$ kleiner als $\mathrm{P}(E)$ ist. Wenn $\mathrm{P}(E \mid H)$ und $(E)$ gleich groß sind, dann ist die Beobachtungsaussage unabhängig von der Hypothese, und es gilt $\mathrm{P}(H \mid E)=\mathrm{P}(H)$, d.h. die Beobachtung hat keinen Einfluss auf die Wahrscheinlichkeit der Hypothese.

Des Weiteren hängt die Endwahrscheinlichkeit im Bayes'schen Theorem von den drei Termen der rechten Seite ab. Wenn wir jeweils einen Term variieren, und die beiden anderen Terme festhalten, dann ergeben sich drei einfache Proportionalitäten (vgl. etwa Howson/Urbach 1993, S. 80). Erstens ist die Endwahrscheinlichkeit $\mathrm{P}(H \mid E)$ einer Hypothese proportional zur Anfangswahrscheinlichkeit $\mathrm{P}(H)$ : Je größer die Wahrscheinlichkeit der Hypothese vor der Beobachtung schon war, desto größer ist sie auch hinterher. Das leuchtet unmittelbar ein. Zweitens ist die Endwahrscheinlichkeit der Hypothese umgekehrt proportional zur Wahrscheinlichkeit der Beobachtung: Je kleiner die Wahrscheinlichkeit der Beobachtung für sich genommen ist, d.h. je überraschender die Beobachtung für das Subjekt ist, desto stärker wird die Hypothese durch die Beobachtung bestätigt.

Drittens ist die Endwahrscheinlichkeit proportional zur Likelihood $\mathrm{P}(E \mid H)$. Auch das ist plausibel. Zwei Extremfälle sind besonders interessant, denn sie liefern zwei Regeln, die zwar sehr einfach, aber trotzdem so wichtig sind, dass zwei komplette Methodologien auf ihnen beruhen, nämlich Poppers Falsifikationismus und die hypothetisch-deduktive Methode. Der erste Extremfall ist, dass die Hypothese $H$ die Falschheit der Beobachtungsaussage $E$ vorhersagt, also non- $E$ impliziert. Dann folgt $\mathrm{P}(E \mid H)=0$, und die Endwahrscheinlichkeit $\mathrm{P}(H \mid E)$ ist ebenfalls Null. Die Hypothese macht dann eine falsche Vorhersage, und das rationale Subjekt verwirft die Hypothese nach dem Eintreffen der Beobachtung. Das ist der Kern von Poppers Falsifikationismus. Beim zweiten Extremfall ist die Beobachtungsaussage $E$ eine logische Konsequenz der Hypothese $H$, also $\mathrm{P}(E \mid H)=1$. Dann ist die Stützung, die $H$ durch $E$ erfährt, maximal. Dies ist der Kern der hypothetisch-deduktiven Methode, die von Quine, Hempel und anderen vertreten wurde.

Eine der größten Leistungen des Bayesianismus ist seine systematisierende Kraft. Es gelingt ihm, viele wichtige Intuitionen und Faustregeln über induktives Schließen aus den wenigen Wahrscheinlichkeitsaxiomen herzuleiten und auf diese Weise zu erklären. Was wir eben vorgeführt haben, sind nur die einfachsten Beispiele für solche Erklärungen. Zu den weiteren Leistungen zählt, dass er die bekannten Schwächen anderer induktiver Methoden, wie der hypothetisch-deduktiven Methode, erklären kann, dass er ein nützliches Instrument ist, um Probleme und Paradoxien der Bestätigung wie die Rabenparadoxie oder Goodmans Paradoxie zu untersuchen und dass er sich dazu eignet, historische Episoden aus der Geschichte der Wissenschaft zu analysieren. ${ }^{20}$ Wichtig sind auch die Konvergenztheoreme, die beschreiben sollen, wie sich unsere Meinungen durch das Sammeln von immer mehr Beobachtungen der Wahrheit annähern. ${ }^{21}$

\section{Probleme des Bayesianismus}

Den Erfolgen des Bayesianismus stehen allerdings auch eine ganze Reihe von Problemen gegenüber. So verlange er von rationalen Subjekten die Kenntnis aller logischen und mathematischen Wahrheiten. Das ist eine sehr starke Idealisierung. Eine weitere Idealisierung ist die Annahme, dass die Glaubensgrade präzise reelle Zahlen sind. Eine solche Präzision in unseren Meinungen scheint psychologisch unrealistisch zu sein. Diese und andere Probleme

\footnotetext{
${ }^{20}$ Ein klassisches Beispiel ist Jon Dorlings Analyse des Quine-Duhem-Problems mit Bayes'schen Mitteln (1979). Ein besonders schwieriges aber auch schönes Beispiel ist Patrick Mahers Diskussion von „prediction versus accomodation“ (1988).

${ }^{21}$ Earman (1992).
} 
können wir hier nicht besprechen. Wir beschränken uns auf die Diskussion desjenigen Problems, das dem Bayesianismus nach allgemeiner Wahrnehmung die größten Schwierigkeiten bereitet, nämlich das Problem der Anfangswahrscheinlichkeiten (,,priors“"). Wie sich gleich zeigen wird, eröffnet es die Möglichkeit, den Bayesianismus mit dem Partikularismus zu verbinden.

Das Problem der Anfangswahrscheinlichkeiten besteht in der Frage, woher das Subjekt die Anfangswahrscheinlichkeit der jeweiligen Hypothese, also den Term $\mathrm{P}(\mathrm{H})$ in der Bayes'schen Formel, nehmen soll. Solange das Subjekt nicht weiß, wie es die Anfangswahrscheinlichkeiten bestimmen soll, kann es den Bayesianismus nicht anwenden. Viele Autoren meinen nun, dass die Anfangswahrscheinlichkeiten durch so genannte Plausibilitätsbetrachtungen bestimmt werden können. So schreibt Earman: „In Bayesian terms, [plausibility] arguments are designed to persuade us to assign high priors to some alternatives and low priors to others. (1992, S. 140) Einstein, the consummate master of this art form, appealed to analogies, symmetry considerations, thought experiments, heuristic principles (such as the principle of equivalence), etc. All of these considerations, I am suggesting on behalf of the Bayesians, were deployed to nudge assignments of initial probabilities in favour of the theories Einstein was introducing in the early decades of this century." (1992, S. 197198).

Des Weiteren haben in den letzten Jahren die so genannten Bayes'schen Netze eine stürmische Entwicklung durchgemacht. Wie sich die Bayes'schen Netze dazu eignen, partikularistische Elemente in plausibler Weise zu repräsentieren, wurde von Luc Bovens und Stephan Hartmann eingehend untersucht (2004).

Wesley Salmon präsentiert weitere Beispiele für Plausibilitätsbetrachtungen. Er führt unter anderem Analogien und Einfachheitserwägungen an (1967, S. 125-127). Hier haben wir folglich im Bayesianismus einen Ort für das Bewertungskriterium der Einfachheit, das wir oben beim Partikularismus diskutiert haben. Jedoch ergibt sich sofort das Problem, wie man denn die Plausibilitätsbetrachtungen $\mathrm{zu}$ verstehen hat, insbesondere welchen Status sie innerhalb des Bayesianismus einnehmen. Eine mögliche Weise, wie sie verstanden werden können, ist partikularistisch: Sie lassen sich nicht in allgemeine Regeln fassen, sondern variieren von Situation zu Situation. Das hat zur Folge, dass die Bestimmung der Anfangswahrscheinlichkeiten keine systematische Angelegenheit ist, sondern einen lokalen situationsabhängigen Charakter hat, der keine Verallgemeinerungen zulässt. Die Bezeichnung „Plausibilitätsbetrachtungen“ bedeutet dann nicht, dass ihnen wirklich etwas gemeinsam ist, außer, dass sie zur Bestimmung der Anfangswahrscheinlichkeiten eingesetzt werden. Wenn das stimmt, dann haben wir ein partikularistisches Element im Bayesianismus. Wir haben allerdings keinen kompletten Partikularismus, sondern nur eine „Teil-Partikularisierung“, die nur für die Bestimmung der Anfangswahrscheinlichkeiten gilt; für die Einarbeitung von Daten ist weiter der Bayesianismus zuständig. Doch ist das ein schwerwiegende Beschränkung des Bayesianismus, denn die ursprüngliche Erwartung, dass der Bayesianismus eine umfassende Beschreibung induktiven Schließens liefert, wird nicht erfüllt. ${ }^{22}$

\section{Schluss}

Wie die Partikularisten sind wir beeindruckt von der Vielfalt der induktiven Praktiken in Wissenschaft und Alltag und halten es für eine gewaltige Herausforderung für die

\footnotetext{
${ }^{22}$ Ein weiterer Grund für die Partikularisierung des Bayesianismus, auf den wir mangels Raum nicht eingehen können, ist die Abhängigkeit der Wahrscheinlichkeiten vom Hintergrundwissen, siehe z.B. I.J. Good (1967, S. 322), Earman (1992, S. 70) und Roger Rosenkrantz (1977, S. 33).
} 
Methodologie, eine einheitliche Beschreibung und Begründung für unsere induktiven Praktiken zu formulieren. Der einzige Kandidat für eine einheitliche Theorie wissenschaftlichen Schließens ist heute der Bayesianismus. Doch hat er mit großen Problemen zu kämpfen, die dazu führen, dass er Zugeständnisse an den Partikularismus machen muss. Deswegen erwarten wir nicht, dass er eine vollständige Theorie der Induktion liefern wird, die alle Probleme des induktiven Schließens und der Bestätigung löst. Andererseits gelingt es dem Bayesianismus, viele Aspekte unserer induktiven Praktiken zu erklären und in einen systematischen Zusammenhang zu bringen. Seine systematisierende Kraft müssen auch Partikularisten anerkennen.

\section{Literaturverzeichnis}

Barnes, B. (1982). T.S. Kuhn and Social Science. London: Macmillan.

Barnes, B. und Bloor, D. (1982). "Relativism, Rationalism and the Sociology of Knowledge". In M. Hollis and S. Lukes (eds.), Rationality and Relativism. Cambridge, MA: MIT Press, 21-47.

Bovens, L. und Hartman, S. (2004), Bayesian Epistemology. Oxford University Press.

Brown, H. I. (1988). Rationality. London: Routledge.

Brown, J. R. (2000). "Privatizing the University-the New Tragedy of the Commons." Science, Vol 290, Issue 5497, 1701-1702.

Brown, J. R. (2001). Who Rules in Science?: an Opinionated Guide to the Wars. Cambridge, MA: Harvard Univ. Press.

Carnap, R. (1952, 1962). The Continuum of Inductive Methods. Chicago: University of Chicago Press.

Carnap, R. (1993). Mein Weg in die Philosophie. Stuttgart: Reclam.

Dorling, J. (1979). "Bayesian Personalism, the Methodology of Scientific Research Programs, and Duhem's Problem." Stud. Hist. Phil. Sci., vol. 11, No. 4, S. 341-347.

Earman, J. (1992). Bayes or Bust? A Critical Examination of Bayesian Confirmation Theory. Cambridge, MA: MIT Press.

Feyerabend, P.K. (1975). Against Method..

Feyerabend, P.K. (1978). Science in a Free Society. London: New Left Books.

Fitelson, B. (1999). "The Plurality of Bayesian Measures of Confirmation and the Problem of Measure Sensitivity". In D. Howard (ed), Proceedings of the 1998 Biennial Meetings of the Philosophy of Science Association. 66, S. 362- 378.

Friedman, M. (1998). "On the Sociology of Scientific Knowledge and its Philosophical Agenda". Stud. Hist. Phil. Sci., Vol. 29, No. 2, S. 239-271.

Galison, P. und Stump, D.J. (1996). The Disunity of Science. Boundaries, Contexts, and Power. Stanford: Stanford University Press.

Gillies, D. (1998). "The Duhem Thesis and the Quine Thesis." In M. Curd and J.A. Cover (eds.), Philosophy of Science: The Central Issues. New York; London: Norton, 302-19.

Glymour, C., Spirtes, P. und Scheines, R. (1993). Causation, Prediction and Search. NewYork: Springer Verlag.

Glymour, C. (1998). "What Went Wrong? Reflections on Science by Observation and The Bell Curve." Philosophy of Science 65: 1-32.

Godfrey-Smith, P. (2003). Theory and Reality: An Introduction to the Philosophy of Science. Chicago: University of Chicago Press.

Good, I.J. (1967). "The White Shoe Is a Red Herring." British Journal for the Philosophy of Science 17, S. 322. 
Grünbaum, A. (1988). Die Grundlagen der Psychoanalyse: eine philosophische Kritik. Stuttgart: Reclam.

Hacking, I. (1999). The Social Construction of What?. Cambridge, MA: Harvard Univ. Press. Hempel, C. G. (1966). The Philosophy of Natural Science. Englewood Cliffs: Prentice Hall. Hoyningen-Huene, P. (1989). Die Wissenschaftsphilosophie Thomas S. Kuhns Rekonstruktion und Grundlagenprobleme. Braunschweig: Vieweg.

Howson, C. und Urbach, P. (1993). Scientific Reasoning: The Bayesian Approach. $2^{\text {nd }}$ ed. Open Court Publishing Company.

Horwich, P. (1982). Probability and Evidence. Cambridge: Cambridge University Press.

Hrdy, S. B. (2002). "Empathy, Polyandry, and the Myth of the Coy Female". In Kourany, J. (ed) The Gender of Science. Upper Saddle River, NU: Prentice Hall.

Jeffrey, R.C. (1983). The Logic of Decision. Chicago: University of Chicago Press.

Joyce, J.M. (1998). “A Nonpragmatic Vindication of Probabilism". Philosophy of Science, Vol. 65, No. 4, S. 575-604.

Kitcher, P. (2003). In Mendel's Mirror: Philosophical Reflections on Biology. Oxford: Oxford-Univ-Press.

Kitcher, P. (1987). Vaulting Ambition: Sociobiology and the Quest for Human Nature. Cambridge, MA: MIT Press.

Kitcher, P. (1982). Abusing Science: The Case Against Creationism. Cambridge, MA: MIT Press.

Kitcher, P (2001). Science, Truth, and Democracy. New York: Oxford University Press

Kitcher, P. (2003). In Mendel's Mirror: Philosophical Reflections on Biology. New York: Oxford Univ. Press.

Kitcher, Patricia (1992). Freud's Dream: a Complete Interdisciplinary Science of Mind. Cambridge, MA: MIT Press.

Koertge, N. (2000), “'New age' philosophies of science: constructivism, feminism and postmodernism". The British Journal for the Philosophy of Science, vol. 51, issue 4, 667-683.

Kosso, P. (1992). Reading the Book of Nature. An Introduction to the Philosophy of Science. Cambridge: Cambridge University Press.

Kuhn, T. (1962, 1970). The Structure of Scientific Revolutions. Chicago: University of Chicago Press.

Kuhn, T. (1977). The Essential Tension. Chicago: University of Chicago Press.

Lakatos, I. (1970). "Falsification and the Methodology of Scientific Research Programmes." In I. Lakatos und A. Musgrave (eds.), Criticism and the Growth of Knowledge. Cambridge: Cambridge University Press, 91-196.

Latour, B. und Woolgar, S. (1979). Laboratory Life: the Social Construction of Scientific Facts. Los Angeles: Sage.

Laudan, L. (1984). Science and Values. Berkeley: University of California Press.

Laudan, L. (1996). Beyond Positivism and Relativism. Boulder: Westview.

Lewis, D. (1999). Papers in Metaphysics and Epistemology. Cambridge: Cambridge Univ. Press.

Lipton, P. (1990). Inference to the Best Explanation. London: RKP.

Lipton, P. (2004). "Review of Clark, Peter and Hawley, Katherine (eds.), Philosophy of Science Today". Notre Dame Philosophical Reviews 2004.01.07.

Maher, P. (1988). "Prediction, Accommodation, and the Logic of Discovery." PSA, vol. 1, S. 273-285.

Newton-Smith, W. H. (1981). The rationality of science. Boston: Routledge Kegan Paul.

Nola, R. und Sankey, H. (2000). "A Selective Survey of Theories of Scientific Method". In R. Nola und H. Sankey (eds.), After Popper, Kuhn and Feyerabend. Dordrecht: Kluwer, 1-65. Quine, W.V.O. und Ullian, J.S. (1970). The Web of Belief. New York: Random House. 
Quine, W.V.O. (1986). „Reply to Morton White“. In L. Hahn und P. Schilpp (eds.) The Philosophy of $W$. V. Quine. La Salle, IL: Open Court, S. 663-665.

Rosenkrantz, R. (1977). Inference, Method and Decision. D. Reidel Publishing Company.

Salmon, W. (1967). The Foundations of Scientific Inference. Pittsburgh: University of Pittsburgh Press.

Sankey, H. (1997). Rationality, Relativism and Incommensurability. Aldershot: Ashgate.

Sankey, H. (2000). "Methodological Pluralism, Normative Naturalism and the Realist Aim of Science." In R. Nola und H. Sankey (eds.), After Popper, Kuhn and Feyerabend. Dordrecht: Kluwer.

Sankey, H. (2002). "Realism, Method, and Truth". In M. Marsonet (ed), The problem of realism. Aldershot: Ashgate, 2002.

Shapin, S. und Schaffer, S. (1985). Leviathan and the Air Pump. Cambridge: Cambridge University Press.

Sklar, L. (2000). "Interpreting Theories: The Case of Statistical Mechanics". Brit. J. Phil. Sci. 51, S. 729-742.

Skyrms, B. (2000). Choice and Chance: an Introduction to Inductive Logic. Belmont, Calif.: Wadsworth/Thomson.

Sokal, A. D. (2001). Eleganter Unsinn: wie die Denker der Postmoderne die Wissenschaften missbrauchen. Dt. Taschenbuch-Verlag.

Spohn, W. (2003), "Die Philosophie und die Wissenschaften". Ms. http://www.unikonstanz.de/FuF/Philo/Philosophie/Mitarbeiter/spohn_files/wspohn31.pdf

van Fraassen, B. (1989), Laws and Symmetry, Oxford University Press. 